\title{
Migros Türk - ein (trans-) nationales Unternehmen und Prozesse der Globalisierung im türkischen Lebensmitteleinzelhandel
}

\author{
Alexandra Appel, Martin Franz, Markus Hassler, \\ Marburg
}

\section{Einleitung}

Die Schweizer Einzelhandelsgenossenschaft Migros begann 1954 ihr Engagement in der Türkei und setzte damit den Startpunkt für eine Modernisierung des türkischen Lebensmitteleinzelhandels (LEH). Auch wenn sich die Schweizer Migros 1974 aus derTürkei zurückzog, ist Migros Türk bis heute einer der wichtigsten Akteure im türkischen LEH und inzwischen selbst zu einem transnationalen Unternehmen (Transnational Corporation - TNC) geworden. Das Konzept des transnationalen Unternehmens ist sowohl in der Globalisierungs- als auch in der geographischen Handelsforschung von zentraler Bedeutung. Dabei wird in der schablonenhaften Unterscheidung Zwischen nationalen oder indigenen und transnationalen Unternehmen oft vernachlässigt, dass diese Grenzen durch Prozesse der Globalisierung nicht nur der jeweiligen Branchen der Unternehmen, sondern auch des Finanzsektors längst verschwommen sind.

Der vorliegende Artikel zeichnet am Beispiel von Migros Türk die Geschichte der Internationalisierung des türkischen LEH nach, ordnet diese in globale Prozesse im LEH ein und diskutiert dabei das Konzept des transnationalen Unternehmens. Der Artikel beruht auf 32 qualitativen Experteninterviews, die zwischen Februar und März 2010 sowie März und Juni 2011 in der Türkei durchgeführt wurden. Zu den Interviewpartnern gehörten unter anderem Vertreter verschiedener LEHFormate des Migros Türk-Konzerns und seiner Konkurrenzunternehmen sowie Vertreter von Zulieferern aus der Lebensmittelbranche und von Einzelhandelsverbänden. Im Text sind türkischsprachige Interviewzitate ins Deutsche übertragen worden. Der Artikel ist in vier Teile gegliedert. Teil zwei bietet zunächst eine konzeptionelle Einführung zu TNCs und ihrer Bedeutung bei Prozessen der Globalisierung im Einzelhandel. In Teil drei wird die Entwicklung des Unternehmens Migros Türk analysiert. Im letzten Teil wird ein Fazit gezogen.

\section{Transnationale Unternehmen im Lebensmittel- einzelhandel}

Lebensmitteleinzelhändler wie Carrefour (Frankreich), Tesco (Grossbritannien) und Wal-Mart (USA) haben in den letzten zwanzig Jahren sowohl ihre Filialnetze als auch ihre Zulieferbeziehungen in neue Märkte ausgeweitet und sind so zu TNCs geworden (COE \& Hess 2005). Vereinzelt fanden derartige Prozesse allerdings schon viel früher statt - so begann zum Beispiel Metro (Deutschland) seine ersten Auslandsinvestitionen bereits 1968 (Franz 2011) - ab etwa 1990 gewannen diese Entwicklungen aber schubweise deutlich an Dynamik.

Während Dicken (2011: 110) in seiner Definition von TNCs deren Möglichkeit zur grenzüberschreitenden Machtausübung zum Kriterium für Transnationalität macht, wird der Begriff in diversen Veröffentlichungen zum Einzelhandel (z.B. CoE \& Wrigley 2007; KULKE \& PäTZOLD 2009) für Unternehmen verwendet, die nicht nur grenzüberschreitend Handel betreiben, sondern auch grenzüberschreitend investieren. WRIGLEY et al. (2005: 438) konzeptualisieren TNCs als

«complex configurations of intra-, inter- and extrafirm relational networks. These networks are highly embedded in, and therefore shaped by, the economic, political and institutional contexts of both home and host economies».

Bei der Analyse von Globalisierungsprozessen im Einzelhandel werden die TNCs als Gegenstück zu den einheimischen «indigenous retailers» (COE \& WRIGLEY 2007: 7) gesehen, die unter Umständen nicht nur mit wirtschaftlichen Anpassungen, sondern auch mit politischem Widerstand auf die ausländische Konkurrenz reagieren (FrANZ 2010). Dennoch dominieren mittlerweile im Einzelhandelssektor vieler Staaten Südostasiens, Europas und Lateinamerikas TNCs (COE \& Hess 2005). Die geographische Marktexpansion der dominanten Akteure wird gegenwärtig auch in Afrika und Südasien fortgesetzt. So kommt es, dass auch im Einzelhandel TNCs zu «the primary (movers and shapers〉 of the global economy» (DiCKEN 2011: 109) geworden sind. Aufgrund der besonderen Bedeutung der Einbettung und der meist von produzierenden Unternehmen abweichenden Regulierung für Investitionen, weicht die Organisation von transnationalen Einzelhandelsunternehmen weitgehend von den Strukturen der TNCs im primären oder sekundären Sektor sowie im Dienstleistungsbereich, z.B. bei unternehmensnahen Dienstleistungen oder Offshore-Routinetätigkeiten wie Call Centers, ab (DAwson 2007; WrigLeY et al. 2005).

Die Transnationalisierung erfolgreicher Einzelhändler aus Industriestaaten begann in den 1990er Jahren auf 
Grund weitgehender Konsolidierung und zunehmender Regulierung in den Heimatmärkten sowie günstig verfügbarer Finanzmittel, die in den Ursprungsmärkten der Unternehmen nur noch schwerlich für weitere Expansionen genutzt werden konnten. Als wesentlicher Pull-Faktor, der zur Ausbreitung von Supermärkten weltweit beitrug, kann der Trend zur Liberalisierung und Deregulierung vieler Märkte betrachtet werden (REARDon et al. 2004; Wrigley 2000).

\begin{abstract}
«Besonders interessant sind dabei Länder, die einen raschen wirtschaftlichen Wachstumsprozess durchlaufen und in welchen sich aufgrund steigender Einkommen neue Absatzpotentiale entwickeln [...]» (KULKE 2011: 13).
\end{abstract}

Die Implementierung von Internationalisierungsstrategien im Einzelhandel korreliert häufig mit der Grösse des Unternehmens. Dawson (2007) stellt fest, dass die Unternehmen der zweiten Reihe der Einzelhandels-TNCs - die untere Hälfte der Top 100-Einzelhändler - aktuell deutlich aktiver in neue Märkte expandieren als die grössten TNCs. Bei letzteren ist die Zahl der Staaten, in denen sie vertreten sind, in den letzten Jahren nur noch verhältnismässig wenig gewachsen. Sie scheinen sich darauf zu fokussieren, ihren Marktanteil in den Staaten, in denen sie bereits vertreten sind, zu erhöhen.

Der Markteinstieg von TNCs in einen neuen Markt ist besonders dann erfolgreich, wenn sie

«ein in der Heimat bewährtes Konzept übertragen, das im Zielland noch nicht vorhanden ist und für welches ein Marktpotential besteht» (KuLKE 2011: 13).

Dadurch findet im Zielland häufig ein Formatwandel statt, der mit weiteren Veränderungen verbunden sein kann:Wandel der Stadtbilder und Konsummuster, Veränderungen der Konkurrenzstrukturen und horizontale Konzentrationsprozesse, Innovationen im Bereich der Zuliefernetzwerke und vertikale Integration von Lieferstrukturen durch Einzelhandelsunternehmen (Coe \& Wrigley 2007; Franz 2011; Wortmann 2003).

Reardon und Hopkins (2006) unterscheiden vier raum-zeitliche Wellen der Ausbreitung von Supermärkten und anderen modernen LEH-Formaten. Die erste Welle betraf zwischen Anfang und Mitte der 1990er Jahre die Metropolen in den grossen und/ oder relativ wohlhabenden Staaten Lateinamerikas, die zweite Welle Mittelosteuropa sowie Teile von Ost- und Südostasien. Die dritte Welle erfasste ab Ende der 1990er Jahre Osteuropa, die kleineren und ärmeren Länder Lateinamerikas und Asiens sowie, etwas später, weitere Teile von Südost- und Ostasien. Gleichzeitig expandierten TNCs auch in kleinere Agglomerationen in den Gebieten der ersten Welle.
Die vierte Welle, die Mitte der 2000er Jahren begann, hat Südasien und Westafrika zum Ziel (REARDON et al. 2003; REARdon et al. 2004; REARdON \& HopKINS 2006). Dabei sind diese Wellen der Ausbreitung des modernen LEH nicht an die Expansion von TNCs gebunden: Einheimische Unternehmen übernehmen Neuerungen und tragen damit zur Diffusion neuer Formate und anderer Innovationen - auch in Regionen oder Staaten, in denen keine TNCs aktiv sind - bei. Bei derartigen Wissenstransfers kann zwischen Produktund Prozesswissen unterschieden werden (CURRAH \& Wrigley 2004). Die Sichtbarkeit und Zugänglichkeit der Geschäfte macht das Produktwissen für Konkurrenten leicht kopierbar (DAwson 1994). Deshalb ist das Prozesswissen (z.B. IT-Systeme, logistische Abläufe, Aufbau von Liefernetzwerken) von besonderer strategischer Bedeutung für Einzelhandel-TNCs (Currah \& Wrigley 2004). Um an derartiges Wissen aus einem anderen Unternehmen zu gelangen, gibt es verschiedene Möglichkeiten. Dazu gehören Joint Ventures, Firmenübernahmen, Industriespionage und das Abwerben von Mitarbeitenden. Auch in der Transnationalisierung des Einzelhandels in der Türkei spielen unterschiedliche Methoden des Know-how-Transfers eine entscheidende Rolle (Franz \& Hassler 2011). Am Fallbeispiel des bis vor kurzer Zeit grössten türkischen Lebensmitteleinzelhändlers, Migros Türk, werden im Folgenden die spezifischen Dynamiken in der Türkei analysiert.

\section{Fallbeispiel Migros Türk}

\subsection{Gezielter Know-how-Transfer: die Initialphase ab 1954}

Bereits 1954 wurde der erste «moderne» Lebensmitteleinzelhändler in der Türkei - Migros Türk - gegründet. Mit dem Ziel, die Verteilung von Lebensmitteln zu sichern und zu regulieren sowie das Wachstum des Schwarzmarktes einzudämmen, initiierte die Stadtverwaltung Istanbuls die Gründung eines Joint-Ventures mit der Schweizer Migros Genossenschaft. Insgesamt investierten 19 Teilhaber in das neue Unternehmen. Der Grossteil wurde allerdings aus Krediten der staatlichen Ziraat Bank (Landwirtschaftsbank) und der Yapı Kredi Bank, der ersten privaten Bank der Türkei, finanziert (Özcan 2007: 189). Damit waren Akteure, die sonst auf sehr unterschiedlichen Massstabsebenen aktiv sind, in die Gründung des Unternehmens involviert: von lokalen Akteuren wie der Stadtverwaltung Istanbul, nationalen Akteuren wie Banken bis hin zur Schweizer Migros, die damit zum ersten Mal in ihrer Geschichte zum TNC wurde. Hier zeigt sich auch, dass in dieser ersten Welle der Internationalisierung und Modernisierung des LEH in der Türkei nicht die von WrIGLEY (2000) für die Internationalisierungsschübe der 1990er Jahre festgestellten Faktoren eine Rolle 
spielten. Vielmehr war es eher das aktive Werben um einen ausländischen Einzelhändler durch staatliche Institutionen, um gezielt sowohl Produkt- als auch Prozesswissen über modernen LEH in die Türkei zu übertragen.

Bis 1957 operierte Migros Türk nach dem ursprünglichen helvetischen Vorbild mit mobilen Einkaufslastwagen, die Lebensmittel zu bestimmten Uhrzeiten an bestimmten Orten innerhalb Istanbuls anboten. 1959 hatte Migros Türk bereits acht Selbstbedienungsläden, elf Strassenstände und sechzig Verkaufswagen (ÖZCAN 2007: 190). Die Gründung von Migros Türk unter Beteiligung der Stadt Istanbul wurde zum Vorbild für andere Städte in der Türkei, und so folgten auch die Städte Ankara und Izmir mit der Gründung staatlicher Supermärkte. In Ankara wurde 1956 der erste GimaMarkt eröffnet und die ersten Tansaş-Märkte wurden 1973 in Izmir eröffnet. Daneben schaffte das Militär ab 1963 die Ordu-Pazarları-Supermärkte (OYAK CORPORATE 2011).

Zwar war Migros Türk anfänglich erfolgreich, steigende Inflationsraten und eine hohe Staatsverschuldung blieben allerdings problematisch für die Schweizer Investoren. Die grössten Probleme ergaben sich aus den staatlichen Preiskontrollen, dem Mangel an Fachkräften und dem hohen Aufwand zur Instandhaltung der mobilen Verkaufswagen, insbesondere wegen der Import-Regelungen und dem Mangel an Ersatzteilen (ÖZcan 2007). Im Jahr 1960 wurde Migros Türk einvernehmlich umstrukturiert: Die Schweizer Migros Genossenschaft übernahm gemeinsam mit staatlichen Instanzen, darunter die Istanbuler Stadtverwaltung, die Et Balık Kurumu (Gesellschaft für Fleisch und Fisch) sowie die Ziraat Bank, 51\% der Anteile. Damit wurde Migros Türk zu einem quasi-staatlichen Unternehmen (ÖZCAN 2007: 191) (Abb. 1).

\subsection{Anfänge der Liberalisierung und Deregulierung 1975-1989}

Die Jahrzehnte zwischen 1960 und 1980 waren von wirtschaftlicher Instabilität gekennzeichnet. Dazu kamen die Ölkrisen der 1970er Jahre, die die hohe Arbeitslosigkeit und die Inflationsraten verstärkten. Ausserdem herrschten politische Instabilität und die Angst vor, dass traditionelle und islamische Kräfte die laizistische Staatsordnung unterlaufen könnten. Aus diesen Gründen kam es 1960, 1971 und $1980 \mathrm{zu}$ Militärputschen in der Türkei. Diese Entwicklungen trugen dazu bei, dass der Schweizer Teilhaber 1975 seine Anteile an die Koç Holding, eine der grossen türkischen Wirtschaftskonglomerate, verkaufte. Damit wurde Migros Türk zu einem rein türkischen Unternehmen. Den Namen «Migros» durfte das Unternehmen weiterhin verwenden - allerdings nur in der Türkei. Mit der Schweizer Migros Genossenschaft bestehen heute, neben den Ausgleichszahlungen für die Namensverwendung, vorwiegend informelle Verknüpfungen:

\begin{abstract}
«We only use their name. And I think we pay some loyality because we are using their name. [...] But that's the only relation with them. [...] From time to time they want to come to Turkey and we take them and show our locations to them. But we don't have to and they don't have to. But we do it since we are used to have a link some years ago» (Interview mit Supply-chain-solutions Manager von Migros Türk, 2011).
\end{abstract}

Mit dem Militärputsch 1980 wurden auch die Liberalisierungs-, Deregulierungs- und Privatisierungsmassnahmen vorangetrieben. Eine Umstellung des Wirtschaftssystems war ausserdem als Auflage der Strukturanpassungsprogramme des Internationalen Währungsfonds (IWF) im Rahmen der finanziellen Hilfestellung nach dem letzten Militärputsch verbindlich. Die auf Import-Substitution basierende Wirtschaft wurde schrittweise bis 1988 in eine liberale Marktwirtschaft umgewandelt. Zudem förderte die türkische Regierung ab 1985 zunehmend den modernen Einzelhandel mit dem Ziel, das Steueraufkommen zu erhöhen (Koç et al. 2008).

Migros Türk expandierte bereits Mitte der 1980er Jahre ausserhalb Istanbuls in Izmir. Bereits seit den Anfängen der wirtschaftlichen Liberalisierungs- und Deregulierungsprozesse zu Beginn der 1980er Jahre entstanden viele türkische Supermarktketten als Kopien der europäischen und nordamerikanischen Vorbilder, die so zur Modernisierung des türkischen LEH beitrugen. Vorwiegend waren sie lokale und regionale Akteure, wie beispielsweise die Ketten Pehlivanoğlu (gegründet 1980), Kiler (gegründet 1981) oder Beğendik (1986).

\subsection{Zunehmende Dynamik ab Anfang der 1990er Jahre}

Echte Dynamik stellte sich allerdings erst Anfang der 1990er Jahre, parallel zu der von REARdon und HopKINS (2006) definierten ersten Welle der weltweiten Supermarktausbreitung, mit dem Markteintritt von Metro und Carrefour und der damit zugespitzten Konkurrenzsituation ein. Ein ehemaliger Einzelhandelsmanager sagte im Interview:

«Der türkische Lebensmitteleinzelhandel ist ganz am Anfang, alles wird sich noch sehr verändern. Sogar sehr am Anfang, die Zeitrechnung beginnt eigentlich erst mit dem Einstieg von Metro und Carrefour».

Metro trat 1990 mit dem Cash \& Carry-Konzept in den türkischen Markt ein. Allerdings eröffneten sie erst 1998 die ersten Real-Hypermärkte. Die ersten erfolg- 


\begin{tabular}{|c|c|c|c|}
\hline $\begin{array}{c}\text { 1. Phase } \\
1954-1975\end{array}$ & $\begin{array}{l}\text { 2. Phase } \\
1975-1996\end{array}$ & $\begin{array}{l}\text { 3. Phase } \\
1996-2008\end{array}$ & $\begin{array}{l}\text { 4. Phase } \\
\text { seit } 2008\end{array}$ \\
\hline $\begin{array}{l}\text { Einführung von Migros in } \\
\text { der Türkei auf Grundlage } \\
\text { staatlicher Initiative }\end{array}$ & $\begin{array}{l}\text { Privatisierung, Expan- } \\
\text { sion \& Innovation von } \\
\text { Migros in der Türkei }\end{array}$ & $\begin{array}{l}\text { Diversifizierung der } \\
\text { Migros-Formate \& } \\
\text { Expansion }\end{array}$ & $\begin{array}{l}\text { Verkauf an britische } \\
\text { Fondsgesellschaft }\end{array}$ \\
\hline $\begin{array}{l}19 \text { Teilhaber: Migros } \\
\text { Schweiz \& staatliche \& } \\
\text { private türkische Akteure }\end{array}$ & $\begin{array}{l}\text { Hauptanteilhaber } \\
\text { Koç Holding }\end{array}$ & $\begin{array}{l}\text { Hauptanteilhaber } \\
\text { Koç Holding }\end{array}$ & $\begin{array}{l}\text { Hauptanteilhaber } \\
\text { BC Partners }\end{array}$ \\
\hline $\begin{array}{l}\text { Transnationales Unterneh- } \\
\text { men, ab } 1960 \text { quasi staatlich }\end{array}$ & $\begin{array}{l}\text { Nationales } \\
\text { Unternehmen }\end{array}$ & $\begin{array}{l}\text { Transnationales } \\
\text { Unternehmen }\end{array}$ & $\begin{array}{l}\text { Transnationales } \\
\text { Unternehmen }\end{array}$ \\
\hline $\begin{array}{l}\text { Versorgung städtischer } \\
\text { Bevölkerung, Regulierung } \\
\text { des Schwarzmarktes in } \\
\text { Istanbul }\end{array}$ & $\begin{array}{l}\text { Ab 1980er Expansionen } \\
\text { in weitere türkische } \\
\text { Provinzen }\end{array}$ & $\begin{array}{l}\text { Expansion in der Türkei } \\
\text { und angrenzenden } \\
\text { Ländern, vorwiegend } \\
\text { im Kaukasus }\end{array}$ & $\begin{array}{l}\text { Konzentration auf } \\
\text { Super- und Hypermärkte, } \\
\text { Verkauf der Discount- } \\
\text { kette Şok }\end{array}$ \\
\hline $\begin{array}{l}\text { Wirtschaftspolitik: } \\
\text { Import-Substitution \& } \\
\text { staatliche Regulierungen }\end{array}$ & $\begin{array}{l}\text { Wirtschaftspolitik: } \\
\text { ab } 1982 \text { Liberalisierung } \\
\text { \& Deregulierung, } \\
\text { FDI-Anreize }\end{array}$ & $\begin{array}{l}\text { Wirtschaftspolitik: } \\
\text { Liberalisierung, } \\
\text { zunehmend Harmoni- } \\
\text { sierung mit EU-Richt- } \\
\text { linien \& IWF-Auflagen, } \\
\text { FDI-Anreize }\end{array}$ & $\begin{array}{l}\text { Wirtschaftspolitik: } \\
\text { Liberalisierung, } \\
\text { FDI-Anreize }\end{array}$ \\
\hline
\end{tabular}

Abb. 1: Phasenmodell der Entwicklung von Migros Türk Phase model of development of Migros Türk

Modèle des phases du développement de Migros Türk Quelle: eigene Darstellung

reichen Hypermärkte führte 1991 Carrefour (seit 1996 aufgrund eines Joint-Ventures mit dem türkischen Wirtschaftskonglomerat Sabancı Holding unter dem Namen CarrefourSA) in der Türkei ein. Seit dem Markteintritt von Metro und Carrefour wurden zahlreiche transnationale, regionale und lokale LEH-Ketten in der Türkei aktiv. Das britische Unternehmen Tesco übernahm 2003 die in Izmir gegründete Supermarktkette Kipa und expandiert seitdem entlang der West- und Südküste. Der spanische Lebensmitteldiscounter Dia eröffnete die ersten türkischen Niederlassungen 1999. Ein Jahr später wurde Dia von Carrefour und in der Türkei vom Joint-Venture aus Carrefour und Sabanci Holding übernommen und agiert seitdem in der Türkei unter dem Namen DiaSA. Carrefour brachte Dia 2011 in Spanien an die Börse. Damit wurde Dia wieder zum eigenständigen Unternehmen mit Sitz in Spanien (CARrefour 2011).

Durch den Markteintritt der TNCs wurde der Formatwandel, hin zu immer grösseren Einzelhandelsformaten, nicht nur beschleunigt, sondern auch deutlich diversifiziert (zielgruppenspezifische Ausdifferenzierung der Formate von Convenience bis Hyper- und Cash \& Carry-Märkte). Die Entwicklungen in der Türkei verliefen allerdings vergleichsweise langsamer als beispielsweise in den grossen und wohlhabenderen Staaten Südamerikas. Während der Anteil der modernen Formate im LEH in den Ländern Brasilien, Chile und Argentinien bereits im Jahr 2002 jeweils über 50\% betrug (REARDON et al. 2007: 3f.), lag dieser Anteil in der Türkei im Jahr 2008 erst bei etwa 32\% (PRICEwaterhouse Coopers Turkey - PWC 2011: 31). Die Gründe dafür werden in der langen Tradition staatlicher Kontrolle gesehen (CoDron et al. 2004).

Neben den Auflagen des IWF beeinflussten auch die Zollunion mit der Europäischen Union seit 1996 und die aufgenommenen Beitrittsverhandlungen seit 1999 die Fortschritte in den Liberalisierungs- und Deregulierungsmassnahmen des türkischen Marktes. Diese wirkten sich auch auf die Attraktivität der Türkei für europäische Investoren aus. Damit wurden in den 1990er Jahren zunehmend die von WrigLEY (2000) 
definierten Pull-Faktoren wirksam, gleichzeitig ist dabei das Engagement des türkischen Staates hervorzuheben. Auch heute noch gibt es starke staatliche Anreize für (ausländische) Investitionen, die Pull-Faktoren für TNCs darstellen: Dazu gehören Steuerbefreiungen oder -begünstigungen, Übernahme von Sozialversicherungsanteilen für Arbeitgeber und Zuteilung von staatlichem Land für die Unternehmensgründung. Gefördert werden sowohl Grossinvestoren als auch kleine und mittelständische Unternehmen. Artikel 3 des Gesetzes 4875 regelt zudem die Gleichstellung ausländischer und türkischer Investoren:

«1. Foreign investors are free to make foreign direct investments in Turkey. 2. Foreign investors shall be subject to equal treatment with domestic investors» (ForEIGN Direct InVestment LaW No. 4875).

Neben den staatlichen Anreizen können ein generelles Wirtschaftswachstum und die dadurch veränderte Einkommensverteilung und -steigerung als wichtigste Pull-Faktoren angesehen werden.

Die 1990er Jahre waren geprägt durch strukturelle Innovationen und räumliche Expansionen im Bereich des LEHs. Ab Mitte der 1990er Jahre nahm die Zahl der grossen Supermärkte mit Verkaufsflächen von mehr als 1.000 Quadratmetern rapide zu (CoDRon et al. 2004: 591). Migros Türk kopierte die in der Schweiz gängige Differenzierung von M-, MM-, und MMMSupermarktformaten. Das M-Format ist ein Nachbarschaftsladen mit kleiner Verkaufsfläche und kleinem Sortiment. Die MMM-Formate verfügen über die grössten Verkaufsflächen und das breiteste und tiefste Sortiment der Migros Türk-Formate. Das Hypermarktformat von Migros Türk heisst 5M. Durch diese Ausdifferenzierung der Formate kam es gleichzeitig $\mathrm{zu}$ einer Spezialisierung (zielgruppenspezifische Orientierung, z.B. Macromarket als Luxus-Supermarktkette) und Despezialisierung (Hypermarktformate, die neben Lebensmitteln z.B. auch Kleidung und Bücher führen) (vgl. WoRTMANn 2003).

Ein wichtiger Meilenstein in der Geschichte des LEH der Türkei ist die Gründung des Discounters BİM 1995 durch türkische, US-amerikanische und saudiarabische Investoren (Koç et al. 2009). «They brought know-how. [...] BIM changed the way of doing things a lot» erklärte die Macromarket-Formatmanagerin von Migros Türk im Interview (2011). BİM ist eine Kopie des deutschen Aldi und der erste Lebensmitteldiscounter in der Türkei (Franz \& Hassler 2011). Als Reaktion auf BİM eröffnete auch Migros Türk eine Discountkette unter dem Namen Şok, wobei sich Migros Türk, ebenso wie BİM, von einem ehemaligen Aldi-Manager beraten liess. So stellen beide Unternehmen heute Beispiele für erfolgreichen Transfer von
Produkt- und Prozesswissen vom TNC Aldi auf die zunächst nationalen Akteure BİM und Şok dar, ohne dass Aldi je selbst in der Türkei aktiv geworden wäre. Damit wurde ein Format eingeführt, das zahlreichen Einkommensgruppen zugänglich ist und sich nicht allein an «small pockets of upper-income consumers in large cities» richtet (REARDON et al. 2007: 3), was vielfach in frühen Arbeiten zur Verbreitung von Supermärkten in Entwicklungs- und Schwellenländern kritisiert wurde (z.B. GoldMAN 1974; Holton 1953).

Auch andere türkische Einzelhandelsunternehmen kopierten zunehmend ausländische Vorbilder, und das teilweise mit grossem Erfolg. Insgesamt gibt es laut Angaben von Perder, dem Verband türkischer Lebensmitteleinzelhandelsketten, mehr als 450 türkische Supermarktketten, die seit Anfang der 1990er Jahre als Reaktion auf die TNCs und das Wachstum von Migros Türk gegründet wurden.

«[...] you can see local brands you have never heard of dominating the market against certain international huge success stories» (Interview mit dem Inhaber eines Zulieferunternehmens, 2011).

Ein Beispiel sind die im thrakischen Teil der Türkei verbreiteten Ardaş-Märkte. Insbesondere DiscountFormate konnten sich gegenüber ausländischen Konkurrenten und Migros Türk profilieren. Neben BİM und Şok ist die 2008 gegründete Kette A101 zu nennen. Heute hat sie 1050 Filialen in 48 Provinzen der Türkei.

1996 wurde Migros Türk selbst zu einem TNC. Unter dem Namen «Ramstore» eröffnete Migros Türk zusammen mit dem Baukonzern Enka Niederlassungen zunächst in Aserbaidschan, dann auch in Kasachstan, Russland, Bulgarien, Mazedonien und Kirgisien. Damit wurde Migros Türk zu einem der sogenannten «smaller second-tier 〈regional〉 retail TNCs» (COE \& WRIGLEY 2007: 3). Andere türkische Einzelhandelsunternehmen wie BIM und Gima folgten dem Vorbild und expandierten ebenfalls ins Ausland (Franz \& HaSsLer 2011).

\subsection{Horizontale Konzentrationsprozesse in den 2000er Jahren}

Die 2000er Jahre wurden von horizontalen Konzentrationsprozessen im LEH der Türkei geprägt. So kam es auch zur Konzentration von Marktmacht. Migros Türk kaufte die ursprünglich in Izmir angesiedelte Kette Tansaş sowie die Supermarktketten Yonca und Maxi. CarrefourSA übernahm die ehemals in Ankara gegründete Kette Gima und die Pınar Marketçilik Supermärkte. Die Kiler Gruppe kaufte die Ketten Canerler, Güler und Karıncalar. Insgesamt wurde der Marktanteil der fünf grössten Akteure im LEH (Migros Türk, CarrefourSA, BİM, Metro, Tesco Kipa), vor dem Verkauf der Migros-Tochter Sok, in der 
Türkei jedoch auf weniger als $20 \%$ geschätzt (PrICEWATERHOUSE COOPERS TuRKey - PWC 2011: 87). Daneben wurden neue Formate erfolgreich etabliert. Dazu gehören high-end-Formate, wie die heutige Migros Türk-Tochter Macrocenter, und Kleinformate im urbanen Raum, wie Migros-Jet als Convenience-Format. Zudem zeichnet sich eine zunehmende vertikale Integration in der Lieferkette von frischem Obst und Gemüse ab (vgl. Koç et al. 2008).

2007 verkaufte Migros Türk ihren Anteil an den knapp achtzig russischen Ramstore-Geschäften an Enka, so dass der Baukonzern alleiniger Eigentümer von Ramstore in Russland wurde. Migros Türk blieb aber Eigentümerin der Ramstore-Läden in den anderen genannten Staaten. 2008 verkaufte die Koç Holding ihre Anteile in Höhe von 50,8\% an die britische Fondsgesellschaft BC Partners und trat damit aus dem LEH-Sektor aus. Heute, seit dem Zusammenschluss der ehemaligen Unternehmen Migros Türk T.A.Ş. und Moonlight Perakendecilik ve Ticaret A.Ş. im Jahr 2009, gehören über $80 \%$ der Anteile Migros Ticaret A.Ş. als Tochterunternehmen von BC Partners (Migros Ticaret A.Ş. 2011). Aufgrund der weitgehenden Globalisierung der Finanzmärkte wurde Migros Türk, die in der Türkei nach wie vor als türkisches Unternehmen empfunden wird, Besitz eines britischen Fonds, über den unter anderem auch der türkische Fonds Turkven Private Equity an dem Unternehmen beteiligt ist (BC PARTNERS 2008). Mit der Übernahme durch die britische Fondsgesellschaft BC-Partners werden strategische Entscheidungen für Migros und Ramstore weder in der Türkei noch im Kaukasus getroffen, sondern in Grossbritannien, wo es keine einzige Niederlassung des Migros Ticaret A.Ş. Konzerns gibt.

Die neuen Eigentümerstrukturen wirkten sich auch auf die Strategie aus. Im Mai 2011 wurde das DiscountFormat Şok von Migros Türk an den Lebensmittelhersteller Ülker verkauft.

«[...] it's a strategical decision. They want to focus on the supermarket and hypermarket issue. The discount issue is very different from the supermarket issue. [...] [It's] not a sales operation but a logistics operation»

erklärte ein Şok-Manager im Interview. Dennoch gibt Migros Türk mit dem Verkauf von Şok mehr als die Hälfte ihrer Niederlassungen ab und büsst an Macht gegenüber Zulieferern und Konkurrenten ein. Der Einfluss der Unternehmenskultur wird an diesem Verkauf deutlich. Ein Manager der Supplychain-solutions Abteilung von Migros Türk kommentierte die Transaktion:

«I believe it's in the nature of the operation. I mean every single private equity buys something and sells it, or cuts it apart».

\subsection{Räumliche Dimension der Internationalisierungs- prozesse}

Wie bereits SchröDER (1999) feststellt, können die Auswirkungen der Internationalisierung des Einzelhandels in Abhängigkeit von der räumlichen Analyseebene sowohl räumlich homogenisierend als auch polarisierend interpretiert werden. Die generelle Zunahme an modernen Formaten im LEH in der Türkei verweist demnach auf eine Annäherung der türkischen Marktstruktur an europäische und nordamerikanische Verhältnisse. Der Marktanteil des modernen Einzelhandels am gesamten Einzelhandel in der Türkei beträgt etwa 40 bis 42 Prozent (PricEWaterhouse CoOpers Turkey - PWC 2011: 26). In Europa liegt dieser Marktanteil durchschnittlich zwischen 80 und 90 Prozent. Im Bereich des LEHs wird der Anteil der modernen Formate in der Türkei auf nur 32 Prozent geschätzt (Pricewaterhouse CoOpers TURKEY - PWC 2011:31).

Die räumliche Analyse unter Berücksichtigung einzelner Provinzen zeigt allerdings deutliche Konzentrationen in urbanen Räumen. In den eher ländlich geprägten Provinzen Hakkari, Siirt, Tunceli und Şırnak im Südosten der Türkei ist beispielsweise keiner der fünf umsatzstärksten Akteure des LEH (Migros Türk, BİM, CarrefourSA, Metro, Tesco Kipa) der Türkei aktiv, da deren wirtschaftliches Potential, zum Teil auch im Zusammenhang mit dem Kurdenkonflikt, eher gering eingeschätzt wird. In den vier Regionen Zentralanatolien, Schwarzmeerregion, Ostanatolien und Südostanatolien lebt etwa die Hälfte der Einwohner der Türkei. Migros Türk ist in diesen Regionen mit nur 114 ihrer insgesamt $489 \mathrm{M}-$, MM-, und MMM-Niederlassungen vertreten (Migros TicareT A.Ş. 2011). Der Rest der Niederlassungen befindet sich in den wirtschaftlich relativ starken küstennahen Gebieten der Marmararegion, Ägäis und Mittelmeerregion. Discount-Formate sind räumlich generell weiter verbreitet als normale Supermarktketten. So ist Şok, das ehemalige Discountformat von Migros Türk, in nahezu allen Provinzen der Türkei vertreten. Hypermarkt-Formate hingegen gibt es ausschliesslich in urbanen Zentren oder Agglomerationsräumen mit touristischem Potential und/oder einer aufstrebenden Mittelschicht. Macrocenter, das Luxus-SupermarktFormat von Migros Türk, ist beispielsweise ausschliesslich in Istanbul und mit einer Niederlassung in Antalya vertreten und damit räumlich nur punktuell zu finden (Abb. 2).

\section{Fazit}

Die Entwicklung des LEH in der Türkei kann in Bezug auf Prozesse der Globalisierung in vier Phasen unterteilt werden: 


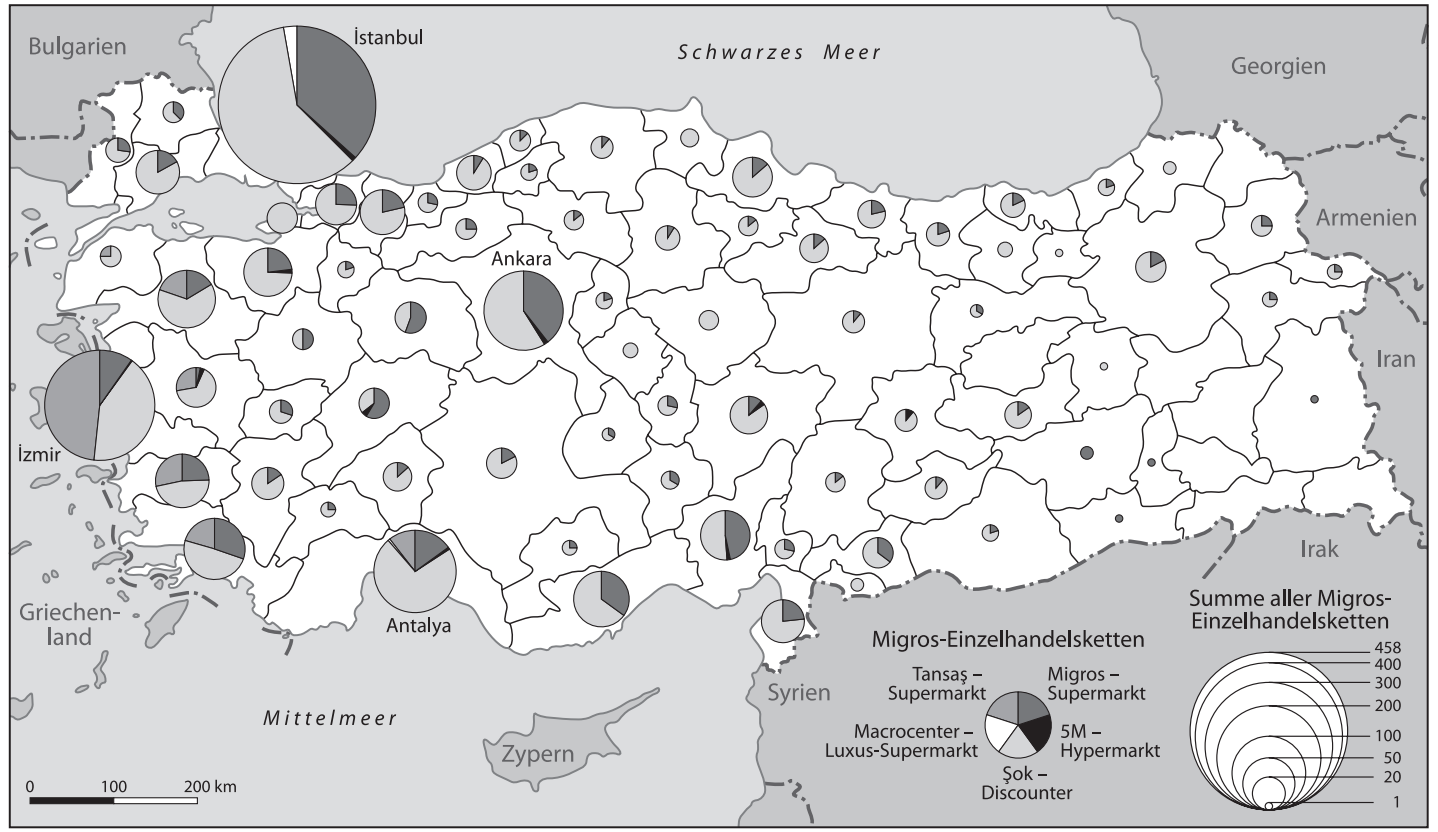

Abb. 2: Räumliche Verteilung der Migros Türk-Formate in der Türkei

Spatial distribution of Migros Türk in Turkey

Distribution spatiale des établissements de Migros Türk en Turquie

Quelle: Migros Ticaret A.Ş. 2011; Kartographie: C. MANN

1. Die Initialphase ab 1954 mit dem Engagement der Schweizer Migros, die deutlich vor der von REARDON und HopkINs (2006) identifizierten ersten Welle der transnationalen Ausbreitung von Einzelhandelsunternehmen stattfand. Ziele waren die effektive und günstige Versorgung der städtischen Bevölkerung und die Kontrolle des Schwarzmarktes. Die von Wrigley (2000) und REARDon et al. (2004) festgestellten Pull-Faktoren spielten in dieser Phase keine Rolle, sondern das aktive Werben um einen ausländischen Einzelhändler durch staatliche Institutionen.

2. Die Jahre 1975 bis 1989 , in denen keine transnationalen Einzelhändler in der Türkei tätig waren und es relativ wenig Dynamik im türkischen LEH-Sektor gab. Die Entwicklungen beschränkten sich auf die Gründung städtischer Supermärkte in weiteren türkischen Agglomerationen nach Migros-Vorbild. Die geringe Dynamik in dieser Phase wurde vielfach auch für andere Entwicklungs- und Schwellenländer beschrieben (z.B. REARDON et al. 2007).

3. Die Phase ab 1990, in der, parallel zu der von REARDON und HopkINS (2006) identifizierten ersten
Welle, diverse transnationale Lebensmitteleinzelhändler in der Türkei aktiv wurden und damit eine ganz neue Marktdynamik schufen. Dabei wurden die ausländischen Vorbilder zunehmend von türkischen Unternehmen kopiert. Sowohl in der steigenden Marktdynamik als auch in der starken Ausprägung von Wissenstransfers nach dem Einstieg von TNCs spiegeln sich Entwicklungen, die auch für andere Märkte festgestellt wurden. Charakteristisch für die Entwicklungen der 1990er Jahre in der Türkei ist die zunehmende Formatdiversifizierung, inklusive der Einführung von Discountern und Hypermärkten. Ab 1996 investierten zudem auch türkische Unternehmen als transnationale Akteure im Ausland. Sie sind Teil der von Dawson (2007) festgestellten starken transnationalen Expansion von Zweite-Reihe-Einzelhandels-TNCs.

4. Im Laufe der 2000er Jahre kam es, ähnlich wie in der von REARDON und HopkINs (2006) beschriebenen dritten Welle, zu einer Ausbreitung von modernen Formaten auch in kleineren Agglomerationen. Gleichzeitig fanden weitreichende horizontale Konzentrationsprozesse statt, wie sie in weiten 
Teilen von Nordamerika und Westeuropa in der zweiten Hälfte des 20. Jahrhunderts ähnlich, aber langsamer stattgefunden haben. Darüber hinaus zeichnen sich vertikale Integrationsprozesse der Zulieferstrukturen ab. Bemerkenswert ist der bereits in der vorangegangenen Phase begonnene Erfolg der Discounter in der Türkei. Hier zeigt sich, dass in Schwellenländern ein grosses Potential für ein Format besteht, das sich nicht auf die mittleren und oberen Einkommensgruppen beschränkt. Dies bestätigt auch die erfolgreiche Expansion der Discountkette BIM in Marokko.

Spezifisch für die Türkei war die Orientierung an dem Schweizer Migros (Genossenschafts-) Modell und dem Ziel, auch die ärmere Bevölkerung zu erreichen. Am Beispiel der Unternehmensgeschichte von Migros Türk spiegelt sich die Dynamik der Globalisierungsprozesse und wirtschaftspolitischen Geschichte der Türkei wider. Vor allem ab den 1990er Jahren spielten dabei Übernahmen, Akquisitionen und Joint-Ventures eine wichtige Rolle. Diese Prozesse gestalten die Identifikation eines Unternehmens mit einem bestimmten Nationalstaat kompliziert, und die Grenzen der analytischen Kategorien verschwimmen. Während manche in der Türkei als transnational empfundene Akteure, z.B. Carrefour SA, DiaSA oder Tesco Kipa, durch Joint-Ventures mit türkischen Unternehmern teilweise weitreichend in der Türkei verankert sind, wird Migros Türk in der Türkei trotz seiner ausländischen Investoren vorwiegend als nationale Einzelhandelsmarke angesehen. Auch die Ramstore-Niederlassungen im Kaukasus machen das Unternehmen heute zum transnationalen Akteur. Der Begriff des TNC sollte daher nicht als statisches Konstrukt verstanden werden. Hervorzuheben ist die dynamische Dimension von TNCs, bedingt durch die beständige Restrukturierung und Anpassung der ihnen zu Grunde liegenden komplexen Netzwerkstrukturen. Aufgrund der zunehmenden Bedeutung moderner Finanzierungsmodelle und der Globalisierung der Finanzmärkte sind die Entscheidungsinstanzen und Eigentumsstrukturen von Einzelhandelsunternehmen immer schwerer zu durchschauen - dies gilt sowohl für den Konsumenten als auch für Wissenschaftler. Das strategische Verhalten eines Unternehmens sollte daher eher in Zusammenhang mit seiner Unternehmenskultur als mit seiner (Trans-) Nationalität gesetzt werden.

\section{Danksagung}

Der Artikel entstand im Rahmen des von der Deutschen Forschungsgemeinschaft (DFG) geförderten Projektes «Internationalisierung des Einzelhandels in der Türkei - Motive, Dynamiken und Auswirkungen».

\section{Literatur}

BC PARTNERS (2008): Migros Turk to be acquired by BC Partners. - www.bcpartners.com/bcp/news/2008/200802-14/ 29.08.2011.

Carrefour (2011): Carrefour acquires 100\% of Dia from its subsidiary Norfin Holder S.L. prior to the distribution of the Dia shares by Carrefour. - Press release, $1^{\text {st }}$ July 2011, www.carrefour.com/docroot/ groupe/C4com/Pieces_jointes/Communiques_financiers/2011/DIACOMMUNIQUE01072011UK.pdf 05.10.2011.

Coe, N.M. \& M. Hess (2005): The internationalization of retailing: implications for supply network restructuring in East Asia and Eastern Europe. - In: Journal of Economic Geography 5, 4: 449-473.

Coe, N.M. \& N. Wrigley (2007): Host economy impacts of transnational retail: the research agenda.In: Journal of Economic Geography 7, 4: 341-371.

Codron, J.M., Boushina, Z., Fort, F., Coudel, E. \& A. Puech (2004): Supermarkets in low-income Mediterranean countries: impacts on horticulture systems. - In: Development Policy Review 22, 5: 587-602.

CuRrah, A. \& N. Wrigley (2004): Networks of organizational learning and adaptation in retail TNCs. - In: Global Networks 4, 1: 1-23.

Dawson, J.A. (1994): Internationalization of retail operations. - In: Journal of Marketing Management 10, 4: 267-282.

DAwson, J.A. (2007): Scoping and conceptualising retailer internationalisation. - In: Journal of Economic Geography 7, 4: 373-397.

Dicken, P. (2011): Global shift - mapping the changing contours of world economy. $-6^{\text {th }}$ edition, London: Sage.

Foreign Direct Investment LaW No. 4875. - In: Resmi Gazete vom 17.06.2003, www.invest.gov.tr/deDE/infocenter/publications/Documents/FDI\%20Law \% 20 in\%20Turkey.pdf 22.07.2011.

FRANZ, M. (2010): The role of resistance in a retail production network: protests against supermarkets in India. - In: Singapore Journal of Tropical Geography 31, 3: 317-329.

Franz, M. (2011): Prozesse der Globalisierung im Einzelhandel - die Akteure und ihre Machtbeziehungen. - In: Geographische Rundschau 63, 5: 4-10.

Franz, M. \& M. HASSLER (2011): Globalisierung durch Supermärkte - transnationale Einzelhändler in der Türkei. - In: Geographische Rundschau 63, 5: 28-34.

Goldman, A. (1974): Outreach of consumers and the modernization of urban food retailing in developing countries. - In: Journal of Marketing 38, 4: 8-16.

Holton, R.H. (1953): Marketing structure and economic development. - In: Quarterly Journal of Economics 67, 3: 344-361.

Koç, A.A., Codron, J.-M., TekelioğLu, Y., Lemeilleur, S., Tozanli, S., Aksoy, Ş., Bignebat, C., Demirer, R. \& N. Mencet (2008): Agrifood sector studies - restruc- 
turing of agrifood chains in Turkey: the produce sector (A). - In: Regoverning markets - small scale producers in modern agrifood markets, www.regoverningmarkets.org/en/filemanager/active?fid=743 05.10.2011.

Koç, A.A., BoluK, G. \& S. KovacI (2009): Concentration in food retailing and anti-competitive practices in Turkey. - Paper prepared for presentation at the $113^{\text {th }}$ EAAE Seminar, September 3-6, 2009, Chania, Crete, Greece.

KULKE, E. (2011): Internationalisierung des Einzelhandels - das Beispiel IKEA. - In: Geographische Rundschau 63, 5: 12-19.

KulKe, E. \& K. PÄTZOLd (2009): Internationalisierung des Einzelhandels - Unternehmensstrategien und Anpassungsmechanismen. - Passau: L.I.S.-Verlag.

Migros Ticaret A.Ş. (2011): Homepage. - www. migroskurumsal.com 29.08.2011.

OYAK CORPORATE (2011): Homepage, corporate history 1961-1970. - www.oyak.com.tr/EN/corporate/corporate-history/1961-1970.html 29.08.2011.

Özcan, G.B. (2007): Surviving through transplantation and cloning: the Swiss Migros hybrid, Migros-Türk. In: MPIfG, 2007: 181-206.

Pricewaterhouse Coopers Turkey - PWC (2011): Shining star: the effects of the retail industry on the Turkish economy. - www.pwc.com/tr/en/publications/industrial/ retail-consumer/published/PwC_AMPD_2010_TR_ Final.pdf 17.12.2011.

Reardon, T. \& R. Hopkins (2006): The supermarket revolution in developing countries: policies to address emerging tensions among supermarkets, suppliers and traditional retailers. - In: The European Journal of Development Research 18, 4: 522-545.

Reardon, T., Timmer, P., Barrett, C. \& J. Berdegué (2003): The rise of supermarkets in Africa, Asia, and Latin America. - In: American Journal of Agricultural Economics 85, 5: 1140-1146.

Reardon,T.,Timmer, P. \& J. Berdegué (2004): The rapid rise of supermarkets in developing countries: induced organizational, institutional, and technological change in agrifood systems. - In: Electronic Journal of Agricultural and Developmental Economics 1, 2: 168-183.

Reardon, T., Henson, S. \& J. Berdegué (2007): «Proactive fast-tracking» diffusion of supermarkets in developing countries: implications for market institutions and trade. - In: Journal of Economic Geography 7, 4:1-33.

SCHRÖDER, F. (1999): Einzelhandelslandschaften in Zeiten der Internationalisierung: Birmingham, Mailand, München. - = Geographische Handelsforschung 3, Passau: L.I.S.-Verlag.

Wortmann, M. (2003): Strukturwandel und Globalisieung des deutschen Einzelhandels. $-=$ WZB Discussion Paper SP III 2003-202a, Berlin:Wissenschaftszentrum Berlin für Sozialforschung.

WrigLEY, N. (2000): The globalization of retail capital: themes for economic geography. - In: Clark, G.L.,
Feldman, M.P. \& M.S. Gertler (Hrsg.): The Oxford handbook of economic geography. - Oxford: Oxford University Press: 292-313.

Wrigley, N., Coe, N.M. \& A. Currah (2005): Globalizing retail: conceptualizing the distribution-based transnational corporation (TNC). - In: Progress in Human Geography 29, 4: 437-457.

\section{Zusammenfassung: Migros Türk - ein (trans-) natio- nales Unternehmen und Prozesse der Globalisierung im türkischen Lebensmitteleinzelhandel}

Die Schweizer Einzelhandelsgenossenschaft Migros wurde 1954 in der Türkei aktiv und löste damit erste Modernisierungsprozesse im türkischen Einzelhandel aus. Bis heute ist Migros Türk - längst losgelöst von den Schweizer Gründern - einer der wichtigsten Akteure im türkischen Einzelhandel und durch Investitionen im Ausland sowie die Übernahme durch eine britische Fondsgesellschaft inzwischen selbst ein transnationales Unternehmen. Mit Hilfe von Experteninterviews zeichnet der Artikel die Unternehmensgeschichte nach und diskutiert anhand der wechselnden, teilweise transnationalen Besitzverhältnisse von Migros Türk das Konzept des transnationalen Unternehmens (Transnational Corporation - TNC). Gezeigt wird, dass TNCs sowohl vor dem Hintergrund des jeweiligen Marktes und seiner wirtschaftspolitischen Entwicklungen als auch im Kontext der jeweiligen Unternehmenskultur betrachtet werden müssen. Prozesse der Restrukturierung und Anpassung sind dabei charakteristisch für die Organisation und Dynamik transnationaler Unternehmen.

Schlüsselwörter: Einzelhandel, Globalisierung, Migros, transnationale Unternehmen, Türkei

\section{Abstract: Migros Türk - a (trans-) national corpora- tion - and processes of globalisation in the Turkish food retail sector}

In 1954, the Swiss retail-cooperative Migros expanded into the Turkish market and initiated the first modernization process within the Turkish retail sector. Until today, the corporate development of Migros Türk has been very dynamic with the divestment of the Swiss parent company to a fully owned Turkish company to a firm owned by a British private equity firm. In addition, Migros Türk followed its own internationalisation strategy into Russia and the Caucasus region. Using in-depth interviews, this article analyses the dynamic corporate history of Migros Türk in relation to the conceptualisation of transnational corporations (TNCs) and strategies developed within the context of the retailing sector. The article shows that retail TNCs have to be studied both against the background of the specific market they are involved in as well as in the context of their distinct corporate cul- 
ture. Processes of restructuring and adaption appear to be characteristic for the peculiar organisation and dynamics of retail TNCs.

Keywords: retail, globalisation, Migros, transnational corporations, Turkey

Résumé: Migros Türk - une entreprise (trans-) nationale: les processus de mondialisation du commerce

\section{d'alimentation de détail en Turquie}

L'arrivée de la coopérative «Migros» en 1954 en Turquie a marqué le début de la modernisation du secteur turc de la vente de détail. Aujourd'hui, «Migros Türk»- depuis longtemps indépendante de ses fondateurs suisses - compte parmi les actionnaires les plus importants des petits commerces turcs. Grâce à ses investissements à l'étranger ainsi qu'à sa reprise par une société de fonds britannique, «Migros Türk» représente une entreprise transnationale. A l'aide d'interviews conduits par des experts, cet article retrace l'historique de cette entreprise et sa transformation en entreprise transnationale. Il montre que les entreprises transnationales telles que «Migros Türk» doivent être analysées en tenant compte à la fois du marché spécifique sur lequel elles interviennent ainsi que de leur culture d'entreprise. Leur processus de restructuration et leur flexibilité sont des caractéristiques importantes de l'organisation et du dynamisme des entreprises transnationales.

Mots-clés: commerce de détail, mondialisation, Migros, entreprises transnationales, Turquie

Dipl.-Geogr. Alexandra Appel, PD Dr. Martin Franz,

Prof. Dr. Markus Hassler, Fachbereich Geographie, Philipps-Universität Marburg, Deutschhausstrasse 10, D-35037 Marburg, Deutschland.

e-mail:

alexandra.appel@staff.uni-marburg.de

martin.franz@staff.uni-marburg.de

markus.hassler@staff.uni-marburg.de

Manuskripteingang/received/manuscrit reçu le 19.10.2011

Annahme zum Druck/accepted for publication/accepté pour publication: 23.1 .2012 